\title{
Sub-micron displacement measurement using a digital image correlation method
}

\author{
Masakazu Uchino \\ Mechanic \& Electronics Research Institute, Fukuoka Industrial Technology Center, Kitakyusyu Japan
}

\begin{abstract}
A high-precise measurement method using a digital image correlation method combined with a fine adjustment stage is proposed for the purpose of the thermal deformation measurement of small parts like the electronic devices. An object mounted on the fine adjustment stage is made to move parallel to the in-plane of the object surface at the displacement magnitude of the sub-micron and the images of the object surface are taken by a CCD camera. Calibration curves of the horizontal or vertical displacement vs. transfer pixel of the every pixel point of image are made by using the obtained images. In the case of disregarding out-of-plane deformation, it is possible to carry out the high-precise in-plane displacement distribution measurement using the calibration curves. As an application, the deformation of a driving electronic package is measured using this system.
\end{abstract}

Key words: Digital image correlation method, Full-field measurement, , Calibration curve, Surface deformation, Sub-micron displacement, Thermal expansion, Electronic device, Sub-pixel accuracy

\section{INTRODUCTION}

In the full-field measurement method, moiré interferometry, holographic interferometry, and speckle interferometry are the high-resolution measurement methods of surface deformation [1,2]. For these methods, a laser light is used as a light source, and there is a problem that it is affected for fluctuation of air of the measurement space. Therefore, it is not suited in the measurement of an object like a heating object, which discharges a heat into the space. While, a digital image correlation method is also one of the full-field measurement methods of surface deformation. This method is not comparatively affected in the fluctuation of air, because a white light is used as a light source and images of an object surface are taken by a CCD camera. Surface deformation is measured by matching from the undeformed image to the deformed image, and magnitude and direction of the deformation of the object surface are simultaneously obtained. In this method, the accuracy of the measurement is dependent on the interpolation method between pixels. It is reported that it can be estimated at the accuracy to \pm 0.02 pixels of the measurement pixel [3]. Therefore, the minimum size of displacement depends on the scale of the measuring object and the number of pixels of a CCD camera.

In this study, for the purpose of the thermal deformation measurement of small parts like the electronic devices, the sub-micron displacement measurement method using a digital image correlation method combined with a fine adjustment stage is proposed. Calibration curves of the horizontal or vertical displacement magnitude vs. transfer pixel of the every pixel point of image are made. Measurement of the sub-micron displacement is performed using these calibration curves. As an application, the deformation of a driving electronic package is measured using this system.

\section{THEORY}

\subsection{Principle of a digital image correlation method [3-5]}

For a digital image correlation method, surface deformation is measured by matching between two images, and magnitude and direction of the deformation of the object surface are obtained. The small subset $(N \times N)$ centered at a pixel point $P(X, Y)$ on the undeformed object image is used to obtain an estimate of the displacement of a point $P$. Intensity values of all points in subsets $(N \times N)$ on the undeformed image are compared to the

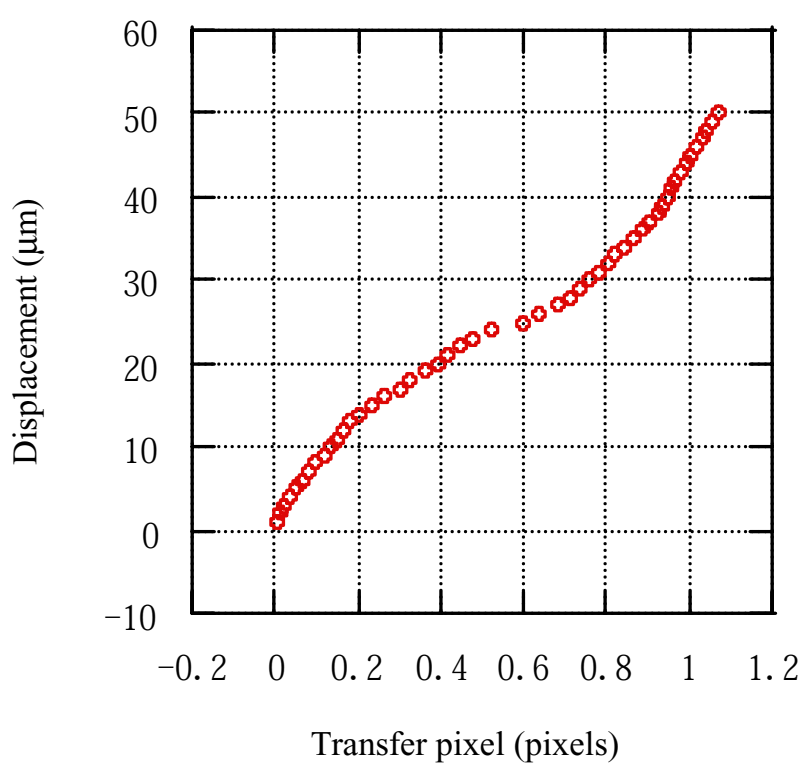

Fig.1. Measurement result of the rigid-body deformation using this system. 
intensity values of other subsets on the deformed image by using the next equation.

$$
\begin{aligned}
& C(X+u, Y+v) \\
& =\sum_{i=-M}^{M} \sum_{j=-M}^{M}\left|I_{d}(X+u+i, Y+v+j)-I_{u}(X+i, Y+j)\right|
\end{aligned}
$$

where $N=2 M+1, C$ is a correlation function. $I_{\mathrm{d}}(X, Y)$ and $I_{\mathrm{u}}(X, Y)$ are intensity values at pixel point $(X, Y)$ on the deformed and undeformed images, respectively. The values of $u$ and $v$ are displacement components in the $\mathrm{x}$ and $\mathrm{y}$ directions, respectively. The values of $u$ ' and $v^{\prime}$, which minimize $C$ in eq.(1) are assumed to be the nearest pixel point $p\left(X^{*}\left(=X+u^{\prime}\right), \quad Y^{*}\left(=Y+v^{\prime}\right)\right)$ transformed from the center of the subset $(N \times N)$ on the undeformed image. The most minimum value of $C$, which is corresponded to the value of displacement less than 1 pixel in the $\mathrm{x}$-direction, will be obtained using a second-order polynomial curve fitting through $C$ of three pixel points $\left(X^{*}-1\right.$ $\left.Y^{*}\right),\left(X^{*}, Y^{*}\right),\left(X^{*}+1, Y^{*}\right)$. While, the value of displacement less than 1 pixel in the y-direction is also obtained using three pixel points $\left(X^{*}, Y^{*}-1\right),\left(X^{*}, Y^{*}\right),\left(X^{*}, Y^{*}+1\right)$. Figure 1 shows the measurement result of rigid-body deformation using this method. Subset size is $61 \times 61$ pixels In Fig. 1, the linearity is not sufficient for the relationship between the displacement magnitude and transfer pixel in the case of small deformation. Therefore, the high-precise digital image correlation method using a calibration curve less than 1 pixel is proposed in order to measure the micro displacement like the thermal deformation.

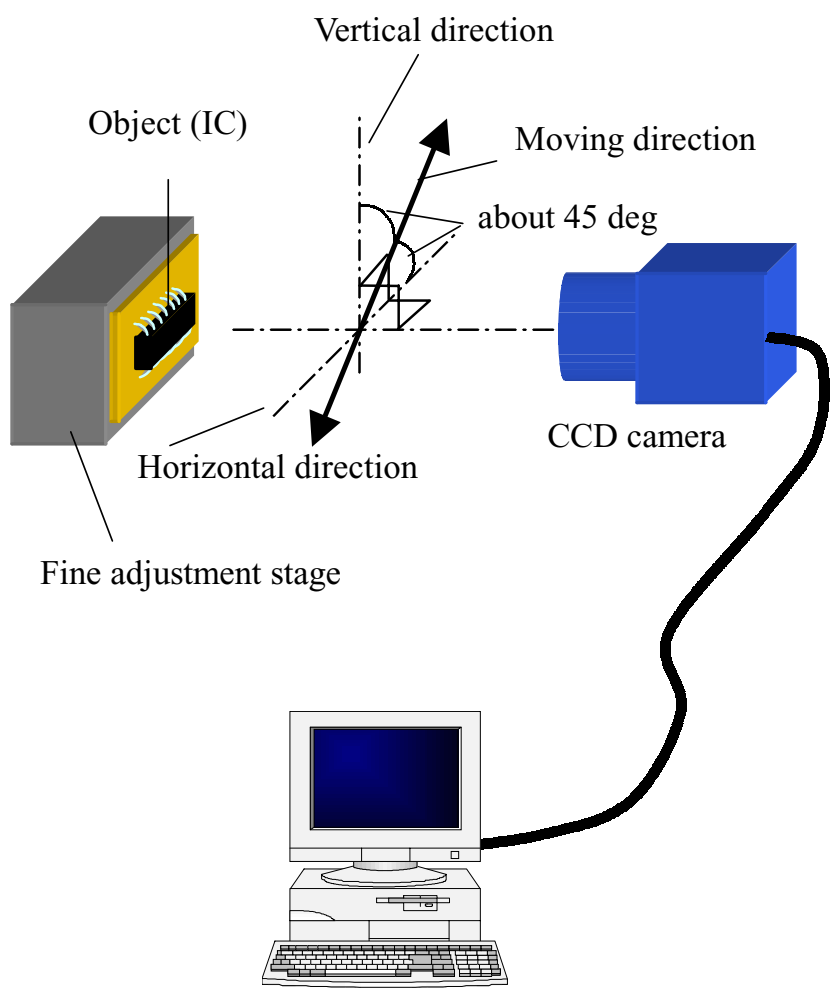

Image processing board, $\&$ analytical computer

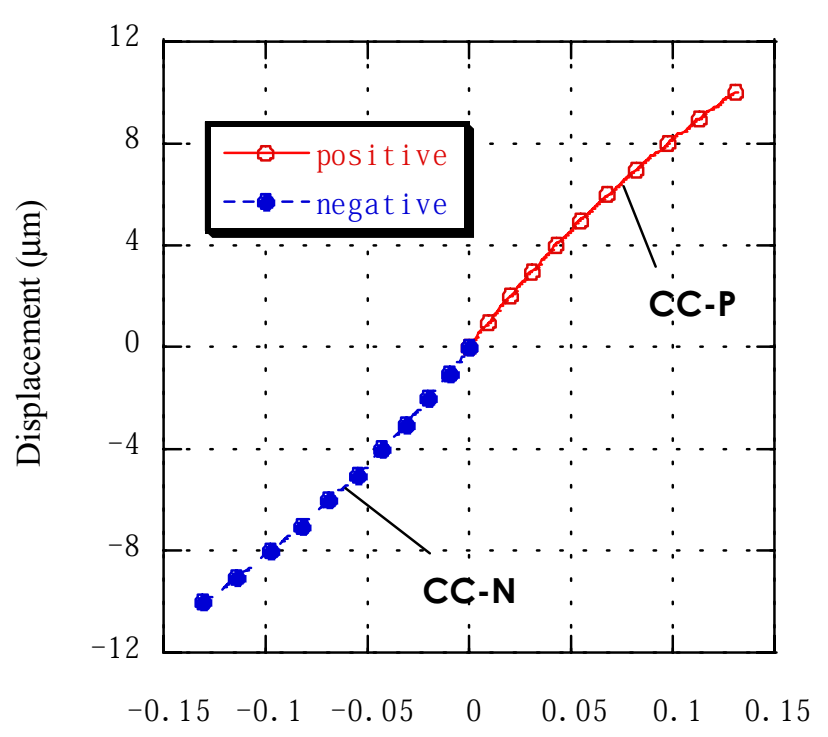

Transfer pixel (pixels)

Fig. 3. Calibration curves of the horizontal direction near the center position of the image.

$$
\begin{aligned}
& \text { CC-P: } y=547.6 x^{3}-287.1 x^{2}+104.7 x+0.0165 \\
& \text { CC-N: } y=488.7 x^{3}+280.6 x^{2}+104.6 x-0.0009
\end{aligned}
$$

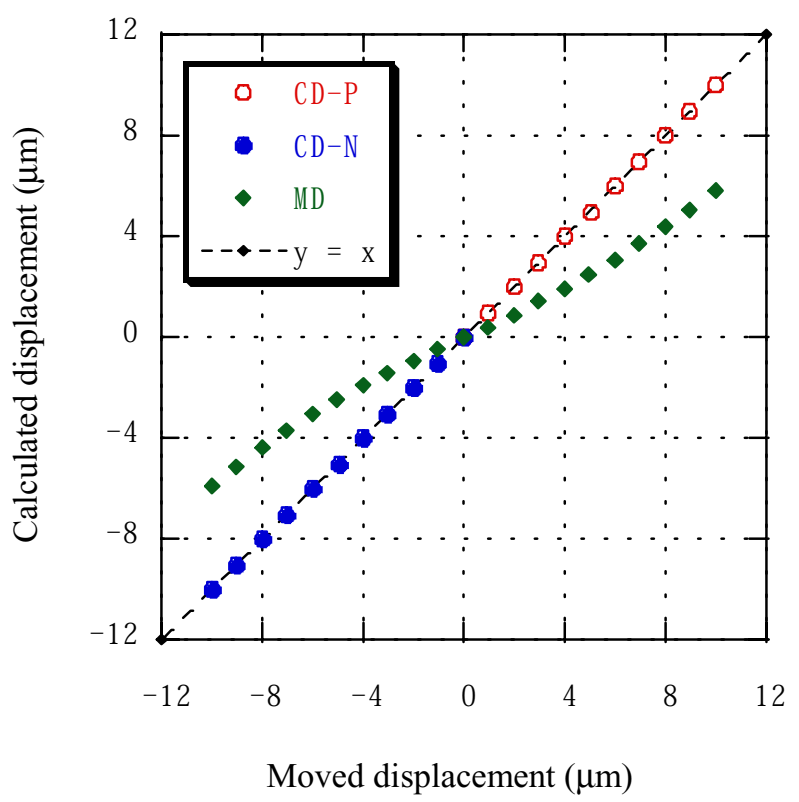

Fig. 4. Calculated displacement using data in Fig. 3 vs. moved displacement. MD: measured data, CD-P, CD-N: converted data using calibration curves in Fig. 3.

Fig.2. Illustration of a digital correlation method using a fine adjustment stage. 


\subsection{Calibration curve}

Figure 2 shows an illustration of the digital image correlation method system for this measurement. This system has been composed of a CCD camera, an image processing board, an analytical computer, and the fine adjustment stage. Random Pattern like a speckle pattern is put on the object surface using white and black color paints. The object mounted on the stage, can be moved at the angle of about $45 \mathrm{deg}$ from the horizontal direction in measurement field. The object is made to move parallel to the in-plane of the object surface at the displacement magnitude of the sub-micron, and the moved image is taken in. This work is repeatedly carried out, and calibration curves of the horizontal or vertical displacement vs. transfer pixel of every pixel point of the image are simultaneously obtained using a third-order polynomial curve fitting.

Figure 3 shows the calibration curves of the horizontal direction in the range of transfer pixel from -0.15 to 0.15 near the center position of the image. Subset size is $61 \times$ 61 pixels, and the displacement magnitude along the horizontal direction is about $44.82 \mu \mathrm{m} / \mathrm{pixel}$. As a result of the analysis, the measured data is not symmetrical for the origin and so two calibration curves of the positive (CC-P) and negative (CC-N) displacement are separately determined, as shown in Fig. 3. For the conversion of the measured data, the calibration curves of the positive and negative displacement are used in the positive and negative data, respectively. Figure 4 shows the difference of the measured data and the converted data using CC-P and $\mathrm{CC}-\mathrm{N}$ in Fig. 3. MD is the measured raw data multiplied by the displacement magnitude $(44.82 \mu \mathrm{m} / \mathrm{pixel})$. CD-P and $\mathrm{CD}-\mathrm{N}$ are converted data using $\mathrm{CC}-\mathrm{P}$ and $\mathrm{CC}-\mathrm{N}$, respectively, and the converted data correspond with the equation $(y=x)$. In the case of disregarding out-of-plane deformation, it is possible to carry out the high-precise in-plane displacement distribution measurement using the calibration curves. In addition, the proofreading of this system is also carried out by this method in being useful.

\section{EXPERIMENTS AND ANALYSIS}

The thermal deformation of the driving electronic package (HD74LS04N: 19x5 $\mathrm{mm}^{2}$ ) is measured using the digital image correlation method and the speckle interferometry method [6]. Figures 5 and 6 are measurement results after drive about 10 seconds of the digital image correlation method and the speckle interferometry method, respectively. The displacement distribution of the measured raw data by the digital image correlation method ( $27 \times 8$ points, every 20 pixels in both direction) is shown in Fig. 5, and an arrow shows the magnitude and direction of the deformation of the point and is magnified 3500 times. In the speckle interferometry method, the measured component of in-plane deformation is parallel to the plane containing two illumination beams and so the Figs. 6 (a) and (b) show the vertical and horizontal displacement components, respectively. In Fig. 6, the image processing is carried out in the speckle fringe pattern, and the contrast of the fringe has been raised. An amount of in-plane deformation for unit fringe spacing is about 0.45 $\mu \mathrm{m}$, and the number in Fig. 6 is correspondent to number of fringes.

The fitting curves of the displacement distribution of

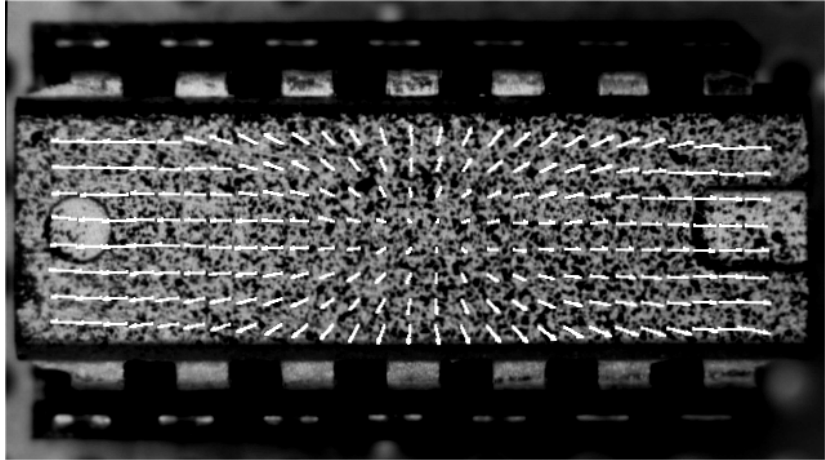

Fig. 5. Measurement results of the displacement distributions using the digital correlation method

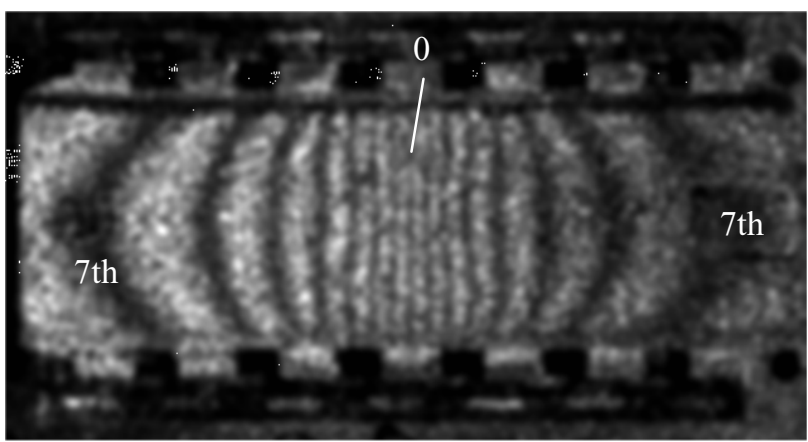

(a)

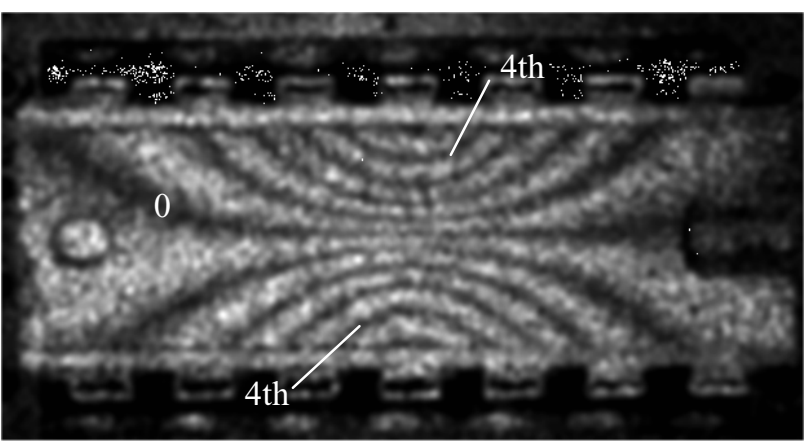

(b)

Fig. 6. Measurement results of the displacement distributions using the speckle interferometry method. (a): horizontal direction, (b) vertical direction

central lines (A-A', B-B') and lower line (C-C') of the electronic device after drive about 10 seconds using the calibration curves are shown in Fig. 7. The displacement magnitudes of thermal expansion of lines of A-A' and B-B' are estimated to be about $7.00 \mu \mathrm{m}$ and $4.14 \mu \mathrm{m}$, re- 
spectively. The displacement magnitudes of horizontal direction of thermal expansion of line of C-C' is estimated to be about $6.90 \mu \mathrm{m}$. In Fig. 7 (a), it is observed that the gradient of the fitting curve around the center part (about 240 pixel to 400 pixel) is larger than other part, and the slope becomes gentle for both outside directions from the center. These results correspond with the distribution of correlation fringe pattern of speckle interferometry method, as shown in Fig. 6 (a), and the gradient of the fitting curve in Fig. 7 (c)-H is also similar. However, the displacement magnitude of the point A in Fig. 7 (a) is large in comparison with the speckle fringe pattern in Fig. 6 (a). As a reason, the error seemed to occur for the analysis of the correlation function, since the contrast of the random pattern around the point A is not sufficient.

While, in Fig. 7 (b), the gradient of the fitting curve corresponds with measurement result of speckle interferometry method, as shown in Fig. 6 (b). Displacement magnitudes of vertical direction of the center point of C-C' is estimated to be about $2.19 \mu \mathrm{m}$, which also corresponds with the speckle fringe pattern in Fig. 6 (b). However, there is a difference at the slopes of the both sides of the fitting curve in comparison with fringe pattern in Fig. 6 (b). As the reason why the difference occurred in the fitting curve, the vertical deformation in the both side parts of the IC package is very small, the analysis of the correlation function is affected for the deformation around the measuring object (i.e. lead of IC).

\section{CONCLUSIONS}

The high-precise displacement measurement method using a digital image correlation method was proposed for the purpose of the thermal deformation measurement of small parts like the electronic devices. Calibration curves of every pixel were obtained using the fine adjustment stage. As an application, the thermal expansion of the driving electronic package was measured using this system, and displacement distribution of the sub micron accuracy is obtained. It is recognized that measuring precision will be improved by using the calibration curves. From now on, calculation of the full-field measurement area should be carried out, and the calculation method (correlation function and calibration curve) will be investigated for establishment of the high precision measurement using the digital image coloration method.

\section{REFERENCES}

1. D. Post, B. Han, and P. Ifju, High Sensitivity Moiré, Springert-Verlag, (1994).

2. R. Jones and C. Wykes, Hologram and Speckle Interferomertry 2nd ed., Cambridge University Press, Cambridge (1989).

3. M. A. Sutton, S. R. McNeill, J. D Helm, and Y. J. Chao, Advances in Two-Dimensional and Three-Dimensional Computer Vision, Springert-Verlag, (2000), pp. 323-372.

4. Z. H. He, M. A. Sutton, W. F. Ranson, and W. H. Peters, Exp. Mech., 24 (1984), pp. 117-121.

5. A. Kato and H. Wate, Proc. APCFS \& ATEM'01, (2001), pp.840-845.

6. M. Uchino, Proc. AFS \& ATEM'01, (2001), pp.836-839. 


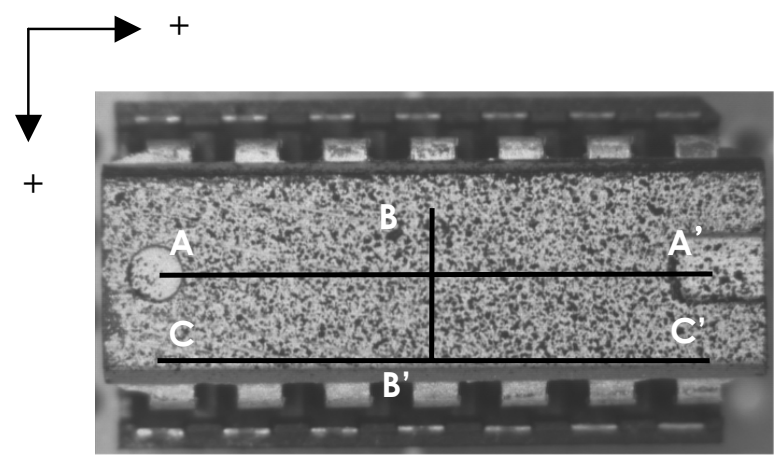

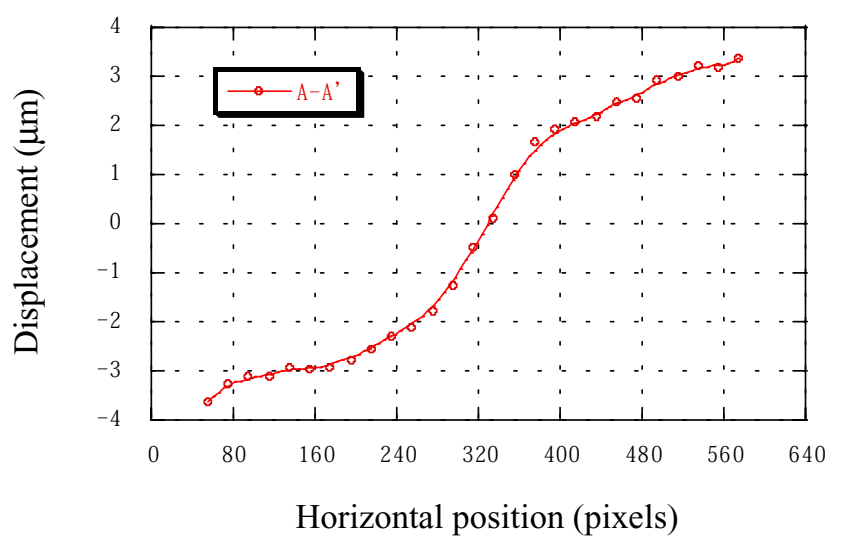

(a)

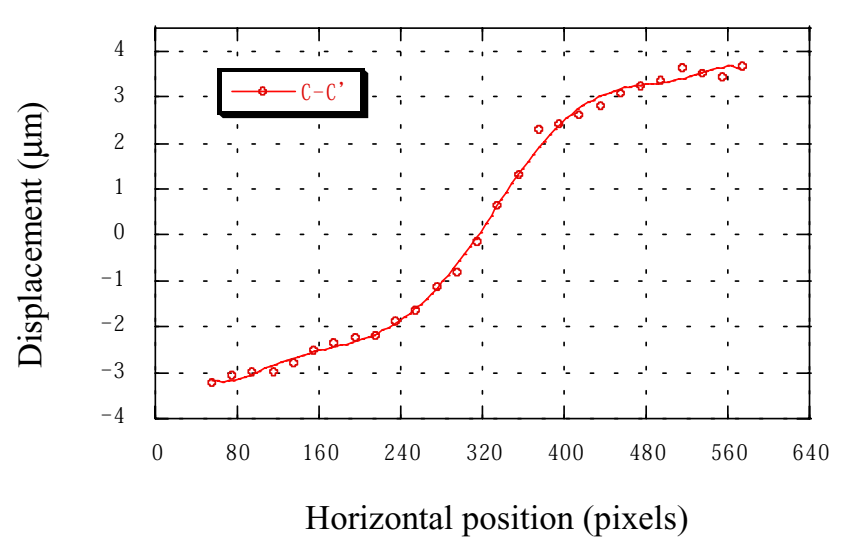

(c)-H

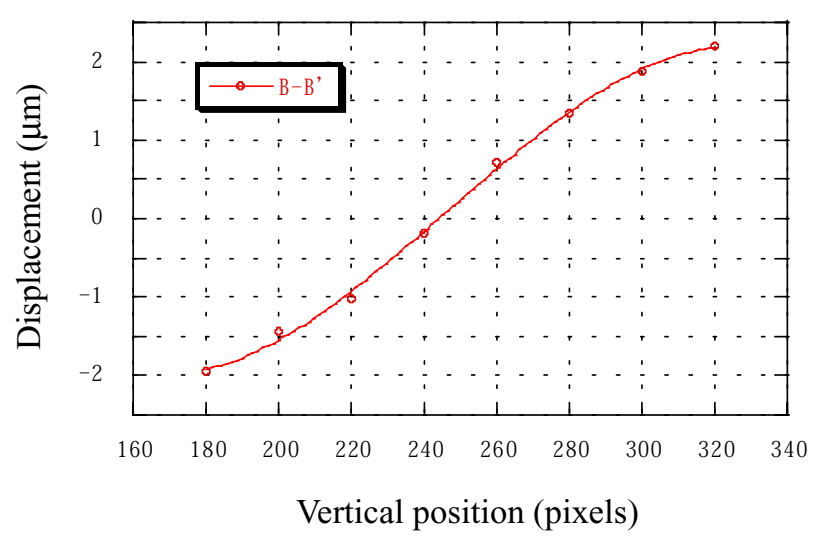

(b)

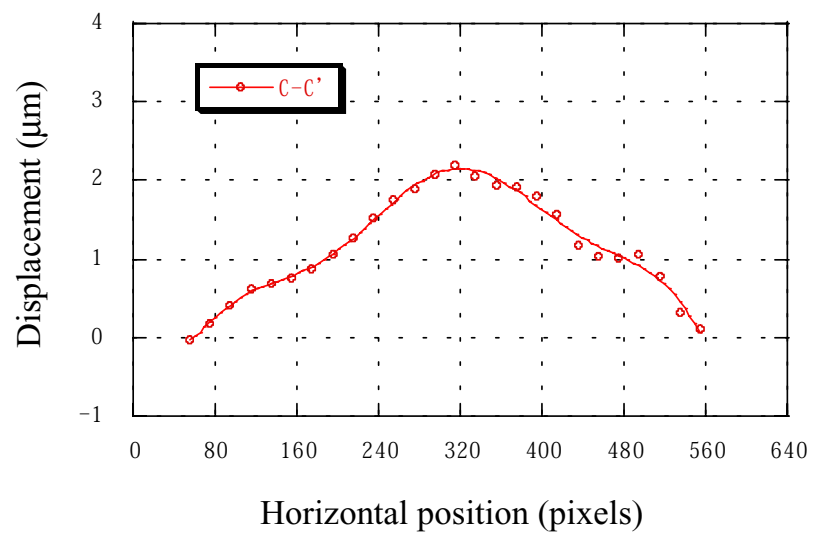

(c)-V

Fig. 7. Results of the displacement distribution of central lines (A-A', B-B') and lower line (C-C') of the electronic device after drive about 10 seconds. (a): horizontal displacement, (b): vertical displacement, (c)-H, (c)-V: horizontal and vertical displacement, respectively. 